\title{
Redes neuronales guiadas con variables internas físicas: cuando la física guía a los datos en el Aprendizaje Profundo.
}

\author{
Jacobo Ayensa-Jiménez ${ }^{1}$, Mohamed H. Doweidar², Manuel Doblaré ${ }^{1}$ \\ ${ }^{1}$ Tissue Microebnvironment Lab (TMELab) \\ Instituto de Investigación en Ingeniería de Aragón (I3A) \\ Universidad de Zaragoza, Mariano Esquillor s/n, 50018, Zaragoza, Spain. \\ ${ }^{2}$ Applied Mechanics and Bioengineering (AMB) \\ Instituto de Investigación en Ingeniería de Aragón (I3A) \\ Universidad de Zaragoza, María de Luna s/n, Edificio Betancourt, 50018, Zaragoza, Spain \\ Tel. +34-976762707, e-mail: jacoboaj@unizar.es
}

\section{Resumen}

Se presenta una metodología hibrida que combina el aprendizaje de las redes neuronales y la incorporación de la física sobre ciertas capas internas de la red. La herramienta tiene poder predictivo y explicativo, mejora a las redes clásicas en velocidad, necesidad de datos, capacidad de filtrado y poder de extrapolación.

\section{Introducción}

Recientemente, se está produciendo un cambio de paradigma en los métodos de simulación computacional. Las técnicas habituales basadas en la formulación de modelos macroscópicos paramétricos que han estado vigentes el último siglo están incorporando cada vez más ingredientes habituales de la Ciencia de Datos. Técnicas como el aprendizaje de variedades [1], búsqueda de vecinos [2,3] o redes neuronales $[4,5]$ se combinan con las ecuaciones de la física del problema para tratar de prescindir de los modelos clásicos utilizados para describir las ecuaciones de estado constitutivas.

No obstante, todos los trabajos hasta la fecha que buscan determinar las ecuaciones de estado del problema están basados en una medida directa de las variables de estado internas. Esto solo es estrictamente posible bajo hipótesis muy particulares $\mathrm{y}$ en ensayos muy controlados. Se presenta una metodología general basada en la medida únicamente de las variables medibles, sin asumir hipótesis adicionales. Esto se consigue identificando las variables de estado internas no medibles con neuronas de ciertas capas ocultas de una red neuronal. Gracias a ello, se consigue una red capaz de, no solo predecir resultados de nuevos experimentos, sino explicar la estructura matemática de las ecuaciones constitutivas.

\section{Métodos}

La metodología consiste en establecer una red neuronal en la que, como en todo problema de ciencia de datos, la entrada sean las variables independientes del problema y la salida sean las variables a predecir. La diferencia con respecto a las redes neuronales habituales radica en que, sobre ciertas capas internas de la red, elegidas estratégicamente, se imponen ciertas restricciones relacionadas con la física más fundamental del problema (leyes de conservación de la masa, momento y energía, así como hipótesis adicionales que se quieran postular). Al tener que satisfacer ciertas relaciones matemáticas prescritas, dichas neuronas adquieren el valor de variables internas con una interpretación física determinada. En términos de las variables de entrada $\boldsymbol{x}$, salida $\boldsymbol{y}$ e internas $\boldsymbol{\eta}$, el problema queda formulado como:

$$
\begin{aligned}
& \boldsymbol{y}=y(x), \\
& \boldsymbol{\eta}=h(\boldsymbol{y}), \\
& \boldsymbol{R}(\boldsymbol{x}, \boldsymbol{y}, \boldsymbol{\eta})=0,
\end{aligned}
$$

donde $y \mathrm{y} h$ son funciones desconocidas que se aprenden mediante Aprendizaje Profundo y $\boldsymbol{R}$ es una función conocida que explicita la física del problema. El proceso de aprendizaje se realiza añadiendo a la función de coste de la red un término de penalización que incluya la función $\boldsymbol{R}$ :

siendo:

$$
L=L_{\text {data }}+L_{\text {phys }}
$$

$$
\begin{aligned}
& L_{\text {data }}=\operatorname{MSE}[\|\widehat{\boldsymbol{y}}-\boldsymbol{y}\|] \\
& L_{\text {phys }}=\operatorname{MSE}\left[\boldsymbol{\Pi}^{\boldsymbol{T}} \boldsymbol{R}(\boldsymbol{x}, \widehat{\boldsymbol{y}}, \widehat{\boldsymbol{\eta}})\right]
\end{aligned}
$$

donde $\hat{\boldsymbol{y}}=y(\boldsymbol{x}), \quad \hat{\boldsymbol{\eta}}=(h \circ y)(\boldsymbol{x})$ y $\boldsymbol{\Pi}$ son metaparámetros adicionales de la red. La función resultante $y$ representa el poder predictivo de la red $\mathrm{y} h$ el poder explicativo. 


\section{Resultados}

El método presentado se ilustra con un problema de hidráulica en el que las ecuaciones constitutivas son complejas y altamente no lineales, pero en el que se conocen algunas leyes fundamentales, como la conservación de la masa y la cantidad de movimiento. En la Fig. 1, se muestra como el método propuesto es capaz de explicar la física subyacente, discriminando entre diferentes modelos constitutivos. En la Fig. 2, se muestra la capacidad predictiva de la red. Además, el método propuesto mejora a las redes neuronales clásicas (sin restricciones) en varios aspectos importantes: convergen más rápido, y necesitan menos volumen de datos, filtran mejor el ruido y extrapolan mejor para predicciones fuera del conjunto de datos de aprendizaje.

\section{Conclusiones}

Uno de los grandes recelos que existe actualmente hacia la inteligencia artificial es que en ocasiones esta funciona como una caja negra. La metodología presentada permite utilizar los importantes desarrollos a nivel de algoritmos (métodos de optimización), software (paralelización, escalabilidad... y y hardware (GPUs y TPUs) en boga

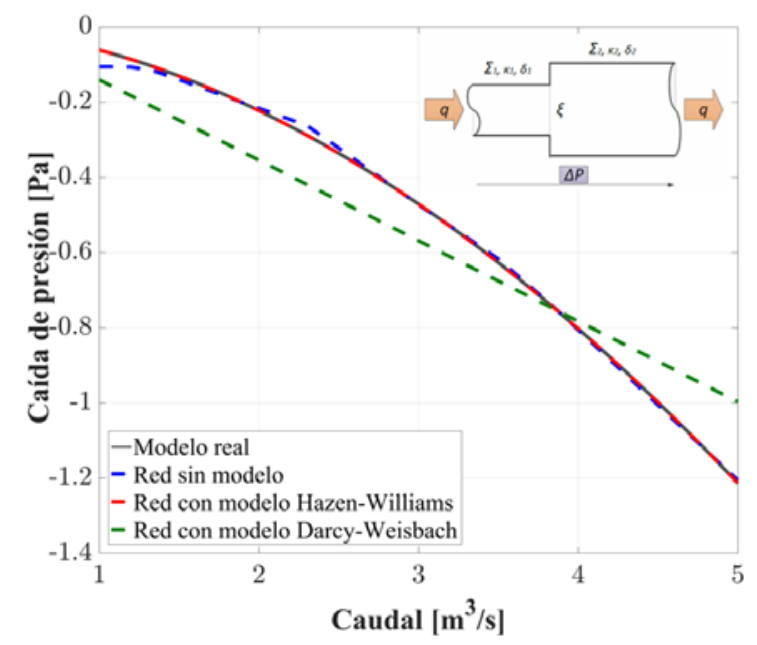

Fig. 1. Predicción de la caída de presión con tres redes neuronales diferentes. La red que incorpora el modelo adecuado (HazenWilliams). Aun así, la red sin modelo es capaz de explicar la física del problema. actualmente sin necesidad de perder de vista la física del problema. Esto permite arrojar luz sobre dicha caja negra, lo que se traduce en una mejor interpretabilidad de todas las variables y en mejoras del rendimiento de la red.

\section{REFERENCIAS}

[1]. BANEZ, Rubén, et al. A manifold learning approach to data-driven computational elasticity and inelasticity. Archives of Computational Methods in Engineering, 2018, vol. 25, no 1, p. 47-57.

[2]. KIRCHDOERFER, Trenton; ORTIZ, Michael. Data-driven computational mechanics. Computer Methods in Applied Mechanics and Engineering, 2016, vol. 304, p. 81-101.

[3]. AYENSA-JIMÉNEZ, Jacobo, et al. A new reliability-based data-driven approach for noisy experimental data with physical constraints. Computer Methods in Applied Mechanics and Engineering, 2018, vol. 328, p. 752-774.

[4]. RAISSI, Maziar; PERDIKARIS, Paris; KARNIADAKIS, George E. Physics-informed neural networks: A deep learning framework for solving forward and inverse problems involving nonlinear partial differential equations. Journal of Computational Physics, 2019, vol. 378, p. 686707.

[5]. HERNANDEZ, Quercus, et al. Structure-preserving neural networks. arXiv preprint arXiv:2004.04653, 2020.

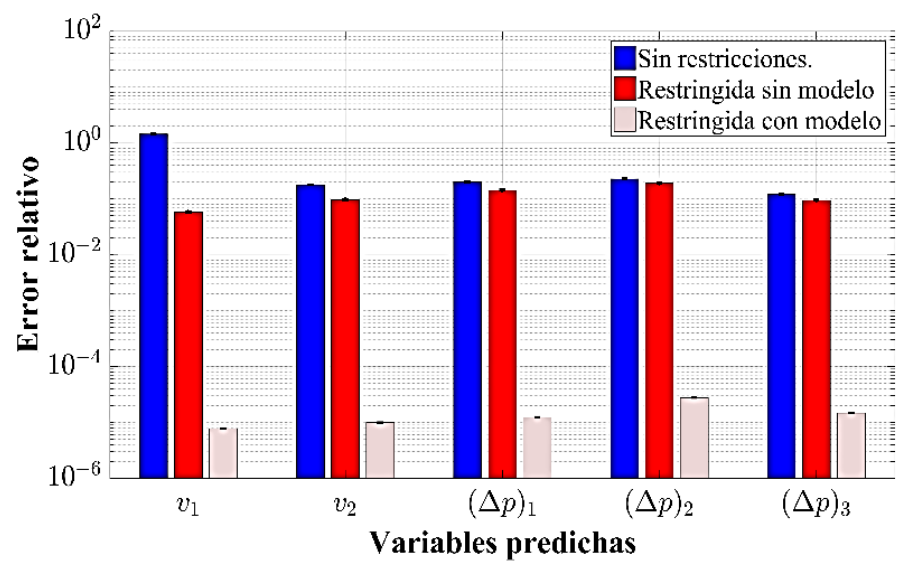

Fig 2. Predicción de todas las variables del problema (medibles y no medibles) para varias redes diferentes. Las redes restringidas mejoran siempre a las redes sin restringir, especialmente en las variables no medibles. 